\title{
A note on the Fisher information matrix of the Birnbaum-Saunders distribution
}

\author{
Artur J. Lemonte \\ Departamento de Estatística, Universidade Federal de Pernambuco \\ Recife, Pernambuco, 50749-540, Brazil \\ arturlemonte@gmail.com
}

Received 8 December 2014

Accepted 5 May 2015

\begin{abstract}
We show that the Fisher information matrix of the Birnbaum-Saunders distribution can present numerical problems for some values of the shape parameter. To overcome this problem, we provide a simple analytical approximation which works very well.

Keywords: Birnbaum-Saunders distribution; Fatigue life distribution; Fisher information matrix; Lifetime data; Fatigue life problems.
\end{abstract}

2000 Mathematics Subject Classification: 60E05

\section{Introduction}

Fatigue is a structural damage which occurs when a material is exposed to stress and tension fluctuations. When the effect of vibrations on material specimens and structures is studied, the first point to be considered is the mechanism that could cause fatigue of these materials. The fatigue process (fatigue life) begins with an imperceptible fissure, the initiation, growth, and propagation of which produces a dominant crack in the specimen due to cyclic patterns of stress, whose ultimate extension causes the rupture or failure of this specimen. The failure occurs when the total extension of the crack exceeds a critical threshold for the first time. The partial extension of a crack produced by fatigue in each cycle is modeled by a random variable which depends on the type of material, the magnitude of the stress, and the number of previous cycles, among other factors.

Motivated by problems of vibration in commercial aircraft that caused fatigue in the materials, $[5,6]$ proposed a family of two-parameter distributions to model failure time due to fatigue under cyclic loading and the assumption that failure follows from the development and growth of a dominant crack. This distribution is known as the two-parameter Birnbaum-Saunders ('BS' for short) distribution or as the fatigue life distribution. The BS distribution is an attractive alternative to the Weibull, gamma, and log-normal models, since its derivation considers the basic characteristics of the fatigue process. It was later derived by [11] using a biological model which followed from relaxing some of the assumptions originally made by [5]. The relationship between the BS distribution and the inverse Gaussian distribution was investigated by [12], who demonstrated that the BS 


\section{A.J. Lemonte}

distribution is an equal-weight mixture of an inverse Gaussian distribution and its complementary reciprocal. For book treatments of inverse Gaussian and BS distributions and their relationships, see [25, Chapter 13] and especially [32, Chapter 10].

The BS distribution has received significant attention over the last few years by many researchers and there has been much theoretical developments with respect to this distribution. The developments have covered many aspects of the distribution. Improved frequentist inference as well as interval estimation are discussed in [22,24] and [34], while Bayes estimation is presented in [2]. Goodness-of-fit tests are considered in [26]. The shape of the hazard function of the BS distribution is discussed in [19], who showed that the hazard function is not monotone and it is unimodal for all ranges of the parameter values. Some generalizations and extensions of the BS distribution are proposed in [7-9,13,15-18,21,29], among others. Other important works about the BS distribution are $[3,4,23]$, among many others.

The BS distribution has also received wide ranging applications. Some recent applications include: modeling of hourly $\mathrm{SO}_{2}$ concentrations at ten monitoring stations located in different zones in Santiago [20]; modeling of diameter at breast height distributions of near-natural complex structure silver fir-European beech forests [30]; modeling of hourly dissolved oxygen (DO) concentrations observed at four monitoring stations located at different areas of Santiago [33]; statistical analysis of redundant systems with one warm stand-by unit [27]. Because of the widespread study and applications of the BS distribution, it is very important to have a Fisher information matrix which works for all the values of the model parameters. In the next section we show that the Fisher information matrix of the BS distribution can present numerical problems for some values of the shape parameter. The aim of this note is to provide a Fisher information matrix which works for all the values of the model parameters of the BS distribution.

\section{The BS distribution}

The cumulative distribution function of a random variable $T$ with BS distribution, say $T \sim$ $\mathrm{BS}(\alpha, \beta)$, is $F(t)=\Phi(v)$, with $t>0$, where $\Phi(\cdot)$ is the standard normal cumulative function, $v=v(t)=\rho(t / \beta) / \alpha, \rho(z)=z^{1 / 2}-z^{-1 / 2}$, and $\alpha>0$ and $\beta>0$ are the shape and scale parameters, respectively. The scale parameter is also the median of the distribution, since $F(\beta)=1 / 2$. For any $k>0$, it follows that $k T \sim \mathrm{BS}(\alpha, k \beta)$. It is noteworthy that the reciprocal property holds for the BS distribution: $T^{-1} \sim \mathrm{BS}\left(\alpha, \beta^{-1}\right)$. The probability density function of $T \sim \mathrm{BS}(\alpha, \beta)$ is $f(t)=$ $\kappa(\alpha, \beta) t^{-3 / 2}(t+\beta) \exp \left[-\tau(t / \beta) /\left(2 \alpha^{2}\right)\right]$, where $\kappa(\alpha, \beta)=\exp \left(\alpha^{-2}\right) /(2 \alpha \sqrt{2 \pi \beta})$ and $\tau(z)=z+$ $z^{-1}$. The BS density function is positively skewed and the asymmetry of the distribution decreases with $\alpha$. As $\alpha$ decreases, the distribution becomes more symmetric around $\beta$, the median. The expected value, variance, skewness and kurtosis are, respectively, $\mathrm{E}(T)=\beta\left(1+\alpha^{2} / 2\right), \operatorname{var}(T)=$ $(\alpha \beta)^{2}\left(1+5 \alpha^{2} / 4\right), \mu_{3}=16 \alpha^{2}\left(11 \alpha^{2}+6\right) /\left(5 \alpha^{2}+4\right)^{3}$ and $\mu_{4}=3+6 \alpha^{2}\left(93 \alpha^{2}+41\right) /\left(5 \alpha^{2}+4\right)^{2}$.

The log-likelihood function $\ell(\alpha, \beta)$ for a single observation $t$ of $T \sim \mathrm{BS}(\alpha, \beta)$, except for a constant term, is given by $\ell(\alpha, \beta)=\log [\kappa(\alpha, \beta)]+\log (t+\beta)-\tau(t / \beta) /\left(2 \alpha^{2}\right)$. By taking partial derivatives of $\ell(\alpha, \beta)$ with respect to $\alpha$ and $\beta$, we obtain the unit score functions: $\partial \ell(\alpha, \beta) / \partial \alpha=$ $-\left(1+2 \alpha^{-2}\right) / \alpha+\tau(t / \beta) / \alpha^{3}$ and $\partial \ell(\alpha, \beta) / \partial \beta=-1 /(2 \beta)+(t+\beta)^{-1}+\rho\left(t^{2} / \beta^{2}\right) /\left(2 \alpha^{2} \beta\right)$. For interval estimation and hypotheses test inference on the model parameters, we require the $2 \times 2$ per observation expected information matrix $K(\alpha, \beta)$, namely

$$
K(\alpha, \beta)=\left[\begin{array}{ll}
K_{\alpha \alpha} & K_{\alpha \beta} \\
K_{\beta \alpha} & K_{\beta \beta}
\end{array}\right]
$$


whose elements are

$$
K_{\alpha \alpha}=\frac{2}{\alpha^{2}}, \quad K_{\alpha \beta}=K_{\beta \alpha}=0, \quad K_{\beta \beta}=\frac{1+\alpha(2 \pi)^{-1 / 2} h(\alpha)}{(\alpha \beta)^{2}},
$$

where

$$
h(\alpha)=\alpha\left(\frac{\pi}{2}\right)^{1 / 2}-\pi \mathrm{e}^{2 / \alpha^{2}}\left[1-\Phi\left(\frac{2}{\alpha}\right)\right] .
$$

Note that $K_{\alpha \beta}=K_{\beta \alpha}=0$ and hence $\alpha$ and $\beta$ are orthogonal [10]. The algebraic development to obtain $K(\alpha, \beta)$ is provided in the Appendix.

In what follows, we show that $K_{\beta \beta}$ (i.e. the function $h(\alpha)$ ) can present some numerical problems for small values of the shape parameter $\alpha$. A simple R function [31] to compute $h(\alpha)$ is given in the Appendix. Table 1 lists some values of $h(\alpha)$ obtained by using the $\mathrm{R}$ function provided in the Appendix. From the figures in Table 1, it is evident that the function $h(\alpha)$ presents numerical problems for small values of $\alpha$, once it delivers $\mathrm{NaN}$ (Not a Number) for small values of the shape parameter $\alpha$. It happens because the product

$$
\mathrm{e}^{2 / \alpha^{2}} \times\left[1-\Phi\left(\frac{2}{\alpha}\right)\right]
$$

delivers an undefined real number for small values of $\alpha$; that is, for a small value of $\alpha$, we have that $\mathrm{e}^{2 / \alpha^{2}}=\infty$, whereas $1-\Phi(2 / \alpha)=0$. So, zero times infinity is an undefined real number. Figure 1 plots the function $h(\alpha)$ for $\alpha \in(0,0.3)$. From this figure, notice that $h(\alpha)$ behaves very unstable for $\alpha \in(0,0.3)$. In the next section, we shall derive a simple analytical approximation for $h(\alpha)$ that works very well for small values of the shape parameter $\alpha$.

Table 1. Function $h(\alpha)$ for some values of $\alpha .^{a}$

\begin{tabular}{cc|cc|cc|cc|cc}
\hline$\alpha$ & $h(\alpha)$ & $\alpha$ & $h(\alpha)$ & $\alpha$ & $h(\alpha)$ & $\alpha$ & $h(\alpha)$ & $\alpha$ & $h(\alpha)$ \\
\hline 0.001 & $\mathrm{NaN}$ & 0.013 & $\mathrm{NaN}$ & 0.025 & $\mathrm{NaN}$ & 0.037 & $\mathrm{NaN}$ & 0.049 & $\mathrm{NaN}$ \\
0.002 & $\mathrm{NaN}$ & 0.014 & $\mathrm{NaN}$ & 0.026 & $\mathrm{NaN}$ & 0.038 & $\mathrm{NaN}$ & 0.050 & $\mathrm{NaN}$ \\
0.003 & $\mathrm{NaN}$ & 0.015 & $\mathrm{NaN}$ & 0.027 & $\mathrm{NaN}$ & 0.039 & $\mathrm{NaN}$ & 0.051 & $\mathrm{NaN}$ \\
0.004 & $\mathrm{NaN}$ & 0.016 & $\mathrm{NaN}$ & 0.028 & $\mathrm{NaN}$ & 0.040 & $\mathrm{NaN}$ & 0.052 & $\mathrm{NaN}$ \\
0.005 & $\mathrm{NaN}$ & 0.017 & $\mathrm{NaN}$ & 0.029 & $\mathrm{NaN}$ & 0.041 & $\mathrm{NaN}$ & 0.053 & $\mathrm{NaN}$ \\
0.006 & $\mathrm{NaN}$ & 0.018 & $\mathrm{NaN}$ & 0.030 & $\mathrm{NaN}$ & 0.042 & $\mathrm{NaN}$ & 0.054 & 0.06768 \\
0.007 & $\mathrm{NaN}$ & 0.019 & $\mathrm{NaN}$ & 0.031 & $\mathrm{NaN}$ & 0.043 & $\mathrm{NaN}$ & 0.055 & 0.06893 \\
0.008 & $\mathrm{NaN}$ & 0.020 & $\mathrm{NaN}$ & 0.032 & $\mathrm{NaN}$ & 0.044 & $\mathrm{NaN}$ & 0.056 & 0.07019 \\
0.009 & $\mathrm{NaN}$ & 0.021 & $\mathrm{NaN}$ & 0.033 & $\mathrm{NaN}$ & 0.045 & $\mathrm{NaN}$ & 0.057 & 0.07144 \\
0.010 & $\mathrm{NaN}$ & 0.022 & $\mathrm{NaN}$ & 0.034 & $\mathrm{NaN}$ & 0.046 & $\mathrm{NaN}$ & 0.058 & 0.07269 \\
0.011 & $\mathrm{NaN}$ & 0.023 & $\mathrm{NaN}$ & 0.035 & $\mathrm{NaN}$ & 0.047 & $\mathrm{NaN}$ & 0.059 & 0.07395 \\
0.012 & $\mathrm{NaN}$ & 0.024 & $\mathrm{NaN}$ & 0.036 & $\mathrm{NaN}$ & 0.048 & $\mathrm{NaN}$ & 0.060 & 0.07520 \\
\hline \multicolumn{1}{l}{$\mathrm{NaN}$ Not a Number. }
\end{tabular}

For a random sample $t=\left(t_{1}, \ldots, t_{n}\right)^{\top}$ of size $n$ from the $\mathrm{BS}(\alpha, \beta)$ model, the total log-likelihood function takes the form $\ell_{n}(\alpha, \beta)=\sum_{i=1}^{n} \ell^{(i)}(\alpha, \beta)$, where $\ell^{(i)}(\alpha, \beta)$ is the log-likelihood function for the $i$ th observation $(i=1, \ldots, n)$ as given before. The total score function is $U_{n}(\alpha, \beta)=$ $\left(\sum_{i=1}^{n} \partial \ell^{(i)}(\alpha, \beta) / \partial \alpha, \sum_{i=1}^{n} \partial \ell^{(i)}(\alpha, \beta) / \partial \beta\right)^{\top}$, where $\partial \ell^{(i)}(\alpha, \beta) / \partial \alpha$ and $\partial \ell^{(i)}(\alpha, \beta) / \partial \beta$, for $i=$ $1, \ldots, n$, have the forms given earlier. The total Fisher information matrix is $K_{n}(\alpha, \beta)=n K(\alpha, \beta)$. 


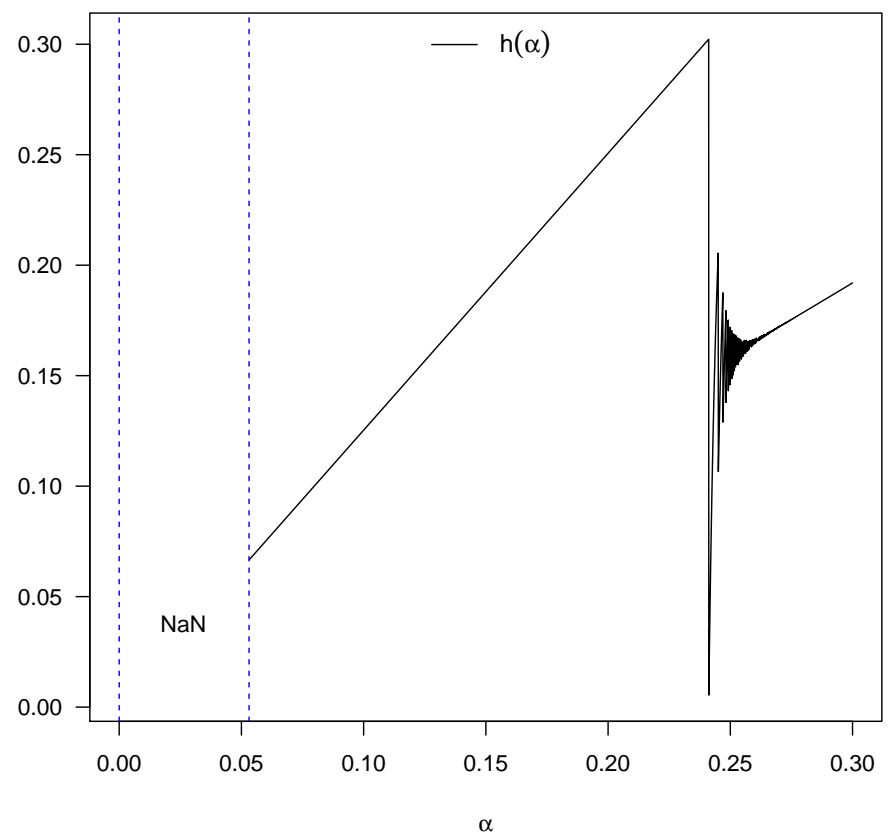

Fig. 1. Function $h(\alpha)$ for $\alpha \in(0,0.3)$.

The maximum likelihood estimates $\widehat{\alpha}$ and $\widehat{\beta}$ of $\alpha$ and $\beta$, respectively, are obtained by setting $U_{n}(\widehat{\alpha}, \widehat{\beta})=0$. There is no closed-form expression for the maximum likelihood estimator and its computation has to be performed numerically using a nonlinear optimization algorithm, such as Newton-Raphson or Fisher scoring algorithms. Under conditions that are fulfilled for parameters in the interior of the parameter space, we have that

$$
\sqrt{n}\left(\begin{array}{c}
\widehat{\alpha} \\
\widehat{\beta}
\end{array}\right) \stackrel{a}{\sim} \mathscr{N}_{2}\left(\left(\begin{array}{l}
\alpha \\
\beta
\end{array}\right),\left[\begin{array}{cc}
\frac{\alpha^{2}}{2} & 0 \\
0 & \frac{(\alpha \beta)^{2}}{1+\alpha(2 \pi)^{-1 / 2} h(\alpha)}
\end{array}\right]\right)
$$

when $n$ is large, “ $\sim$ " denoting approximately distributed. Note that $\widehat{\alpha}$ and $\widehat{\beta}$ are asymptotically independent.

The above asymptotic bivariate normal distribution can be used to construct approximate confidence intervals and confidence regions for the parameters. The asymptotic $100(1-\eta) \%$ confidence intervals for $\alpha$ and $\beta$ are given, respectively, by

$$
\begin{gathered}
{\left[\widehat{\alpha}\left(1-\frac{z_{1-\eta / 2}}{\sqrt{2 n}}\right), \widehat{\alpha}\left(1+\frac{z_{1-\eta / 2}}{\sqrt{2 n}}\right)\right],} \\
{\left[\widehat{\beta}\left(1-\frac{z_{1-\eta / 2} \widehat{\alpha}}{\left\{n\left[1+\widehat{\alpha}(2 \pi)^{-1 / 2} h(\widehat{\alpha})\right]\right\}^{1 / 2}}\right), \widehat{\beta}\left(1+\frac{z_{1-\eta / 2} \widehat{\alpha}}{\left\{n\left[1+\widehat{\alpha}(2 \pi)^{-1 / 2} h(\widehat{\alpha})\right]\right\}^{1 / 2}}\right)\right],} \\
\text { Published by Atlantis Press } \\
\text { Copyright: the authors }
\end{gathered}
$$


where $z_{1-\eta / 2}$ is the quantile $(1-\eta / 2)$ of the standard normal distribution. Notice that $h(\alpha)$ needs to be computed correctly, since the asymptotic variance of $\widehat{\beta}$ (which is used to define the confidence interval for $\beta$ ) depends on it. However, as showed before, this function can present numerical problems for small values of the shape parameter. Therefore, an approximation for $h(\alpha)$ which works for small values of $\alpha$ has to be derived. It will be done in the next section.

\section{A simple approximation for $h(\alpha)$}

First, we have that

$$
\Phi(x)=\frac{1}{2}+\frac{1}{2} \operatorname{erf}(x / \sqrt{2})
$$

where $\operatorname{erf}(\cdot)$ is the error function and it is defined as

$$
\operatorname{erf}(x)=\frac{2}{\sqrt{\pi}} \int_{0}^{x} \mathrm{e}^{-z^{2}} \mathrm{~d} z
$$

It follows that

$$
1-\Phi\left(\frac{2}{\alpha}\right)=\frac{1}{2}-\frac{1}{2} \operatorname{erf}(\sqrt{2} / \alpha)
$$

and hence $h(\alpha)$ can be reduced to

$$
h(\alpha)=\alpha\left(\frac{\pi}{2}\right)^{1 / 2}-\frac{\pi}{2} \mathrm{e}^{2 / \alpha^{2}}[1-\operatorname{erf}(\sqrt{2} / \alpha)] .
$$

For small values of $\alpha$, it can be shown that [1, p. 298]

$$
\mathrm{e}^{2 / \alpha^{2}}[1-\operatorname{erf}(\sqrt{2} / \alpha)]=\frac{\alpha}{\sqrt{2 \pi}}\left(1-\frac{\alpha^{2}}{4}+\frac{3 \alpha^{4}}{16}-\frac{5 \alpha^{6}}{64}\right)+O\left(\alpha^{9}\right)
$$

Therefore, for small values of $\alpha$, the expression for $h(\alpha)$ in (2.1) can be approximated by

$$
h_{1}(\alpha)=\frac{\alpha}{2}\left(\frac{\pi}{2}\right)^{1 / 2}\left(1+\frac{\alpha^{2}}{4}-\frac{3 \alpha^{4}}{16}+\frac{5 \alpha^{6}}{64}\right)+O\left(\alpha^{9}\right) .
$$

Equation (3.1) has a very simple form and it does not depend on any complicated function. It is a simple polynomial in the shape parameter $\alpha$.

In Table 2, we present some values of $h_{1}(\alpha)$ given in equation (3.1) obtained by using the R function provided in the Appendix. We consider exactly the same values for the parameter $\alpha$ of Table 1 . As expected, the figures in Table 2 reveal that no problem happens in computing $h_{1}(\alpha)$ presented in (3.1) for small values of the shape parameter $\alpha$. Figure 2 plots the functions $h(\alpha)$ and $h_{1}(\alpha)$ for $\alpha \in(0,0.3)$. Notice that $h_{1}(\alpha)$ is very stable for $\alpha \in(0,0.3)$, whereas $h(\alpha)$ is very unstable. Therefore, we strongly recommend to use the approximation (3.1) to make inference (confidence intervals and hypothesis testing) on the model parameters of the BS distribution when $\alpha<0.3$.

\section{Acknowledgments}

We gratefully acknowledge grants from CNPq (Brazil) and FACEPE (Pernambuco, Brazil). 
Table 2. Function $h_{1}(\alpha)$ for some values of $\alpha$.

\begin{tabular}{cc|cc|cc|cc|cc}
\hline$\alpha$ & $h_{1}(\alpha)$ & $\alpha$ & $h_{1}(\alpha)$ & $\alpha$ & $h_{1}(\alpha)$ & $\alpha$ & $h_{1}(\alpha)$ & $\alpha$ & $h_{1}(\alpha)$ \\
\hline 0.001 & 0.00063 & 0.013 & 0.00815 & 0.025 & 0.01567 & 0.037 & 0.02319 & 0.049 & 0.03072 \\
0.002 & 0.00125 & 0.014 & 0.00877 & 0.026 & 0.01630 & 0.038 & 0.02382 & 0.050 & 0.03135 \\
0.003 & 0.00188 & 0.015 & 0.00940 & 0.027 & 0.01692 & 0.039 & 0.02445 & 0.051 & 0.03198 \\
0.004 & 0.00251 & 0.016 & 0.01003 & 0.028 & 0.01755 & 0.040 & 0.02508 & 0.052 & 0.03261 \\
0.005 & 0.00313 & 0.017 & 0.01065 & 0.029 & 0.01818 & 0.041 & 0.02570 & 0.053 & 0.03324 \\
0.006 & 0.00376 & 0.018 & 0.01128 & 0.030 & 0.01880 & 0.042 & 0.02633 & 0.054 & 0.03386 \\
0.007 & 0.00439 & 0.019 & 0.01191 & 0.031 & 0.01943 & 0.043 & 0.02696 & 0.055 & 0.03449 \\
0.008 & 0.00501 & 0.020 & 0.01253 & 0.032 & 0.02006 & 0.044 & 0.02759 & 0.056 & 0.03512 \\
0.009 & 0.00564 & 0.021 & 0.01316 & 0.033 & 0.02069 & 0.045 & 0.02821 & 0.057 & 0.03575 \\
0.010 & 0.00627 & 0.022 & 0.01379 & 0.034 & 0.02131 & 0.046 & 0.02884 & 0.058 & 0.03638 \\
0.011 & 0.00689 & 0.023 & 0.01442 & 0.035 & 0.02194 & 0.047 & 0.02947 & 0.059 & 0.03700 \\
0.012 & 0.00752 & 0.024 & 0.01504 & 0.036 & 0.02257 & 0.048 & 0.03010 & 0.060 & 0.03763 \\
\hline
\end{tabular}

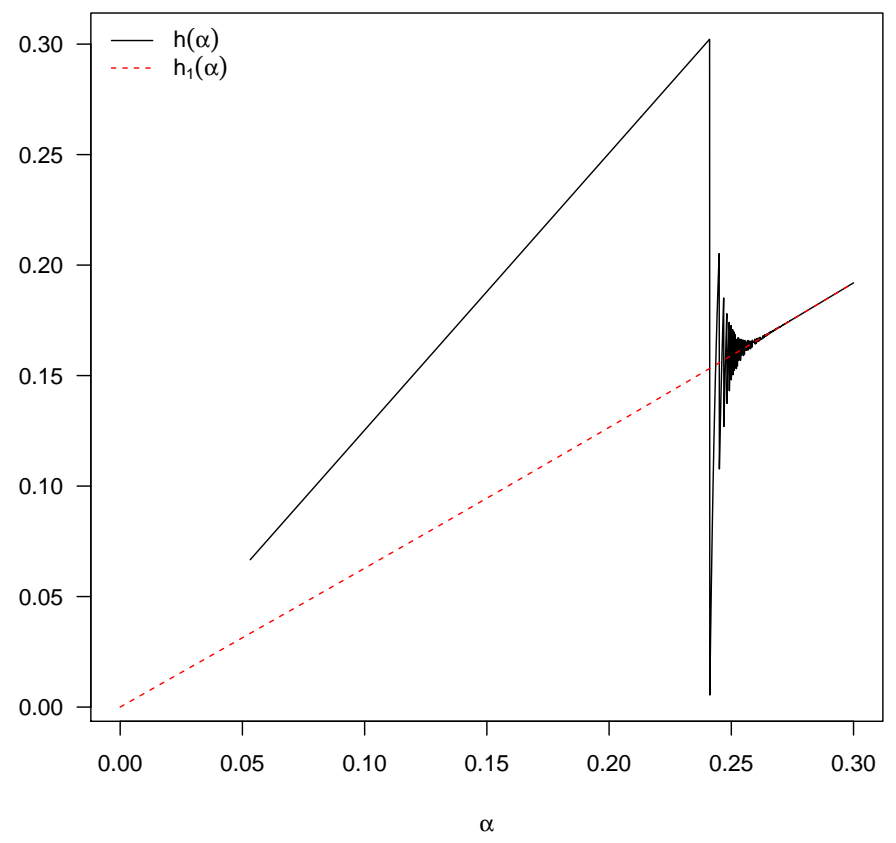

Fig. 2. Functions $h(\alpha)$ and $h_{1}(\alpha)$ for $\alpha \in(0,0.3)$.

\section{Appendix}

It should be mentioned that the asymptotic joint distribution of $\widehat{\alpha}$ and $\widehat{\beta}$ was firstly derived in [14]; see also [28, p. 286]. However, the expression for the Fisher information matrix derived by [14] involves an integral that requires numerical solution, unlike the expression for the Fisher information matrix presented in Section 2, which only involves numerical integration through the evaluation of the standard normal distribution function $\Phi(\cdot)$ (or the error function $\operatorname{erf}(\cdot)$ ). So, the main advantage of using the Fisher information matrix presented in Section 2 over the Fisher information matrix obtained in [14] is that any integral that requires numerical solution has to be computed. 
In the following, we provide a detailed algebraic development to obtain $h(\alpha)$. The second derivatives of the $\log$-likelihood function $\ell(\alpha, \beta)=\log [\kappa(\alpha, \beta)]+\log (t+\beta)-\tau(t / \beta) /\left(2 \alpha^{2}\right)$ are

$$
\begin{gathered}
\frac{\partial^{2} \ell(\alpha, \beta)}{\partial \alpha^{2}}=\frac{1}{\alpha^{2}}+\frac{6}{\alpha^{4}}-\frac{3}{\alpha^{4}}\left(\frac{t}{\beta}+\frac{\beta}{t}\right), \quad \frac{\partial^{2} \ell(\alpha, \beta)}{\partial \alpha \partial \beta}=-\frac{1}{\alpha^{3} \beta}\left(\frac{t}{\beta}-\frac{\beta}{t}\right), \\
\frac{\partial^{2} \ell(\alpha, \beta)}{\partial \beta^{2}}=\frac{1}{2 \beta^{2}}-\frac{1}{(t+\beta)^{2}}-\frac{t}{\alpha^{2} \beta^{3}} .
\end{gathered}
$$

Let $\theta=(\alpha, \beta)^{\top}$ be the parameter vector. Under some regularity conditions, it follows that $K(\alpha, \beta)=$ $-\mathrm{E}\left(\partial^{2} \ell(\alpha, \beta) / \partial \theta \partial \theta^{\top}\right)$. Since $\mathrm{E}(T)=\beta\left(1+\alpha^{2} / 2\right)$ and $\mathrm{E}\left(T^{-1}\right)=\beta^{-1}\left(1+\alpha^{2} / 2\right)$, we have that

$$
\begin{gathered}
K_{\alpha \alpha}=-\mathrm{E}\left(\frac{\partial^{2} \ell(\alpha, \beta)}{\partial \alpha^{2}}\right)=\frac{2}{\alpha^{2}}, \quad K_{\alpha \beta}=K_{\beta \alpha}=-\mathrm{E}\left(\frac{\partial^{2} \ell(\alpha, \beta)}{\partial \alpha \partial \beta}\right)=0, \\
K_{\beta \beta}=-\mathrm{E}\left(\frac{\partial^{2} \ell(\alpha, \beta)}{\partial \beta^{2}}\right)=\frac{1+\alpha^{2} \beta^{2} £_{\beta \beta}}{(\alpha \beta)^{2}},
\end{gathered}
$$

where

$$
£_{\beta \beta}=\frac{1}{2 \alpha \beta \sqrt{2 \pi}} \int_{0}^{\infty} \frac{1}{(t+\beta)^{2}}\left[\left(\frac{\beta}{t}\right)^{1 / 2}+\left(\frac{\beta}{t}\right)^{3 / 2}\right] \exp \left\{-\frac{1}{2 \alpha^{2}}\left(\frac{t}{\beta}+\frac{\beta}{t}-2\right)\right\} \mathrm{d} t .
$$

Making the change of variable $t=\beta u$ in the previous integral, we obtain

$$
£_{\beta \beta}=\frac{1}{2 \alpha \beta^{2} \sqrt{2 \pi}} \int_{0}^{\infty} \frac{u^{-3 / 2}}{(u+1)} \exp \left\{-\frac{1}{2 \alpha^{2}}\left(u^{1 / 2}-u^{-1 / 2}\right)^{2}\right\} \mathrm{d} u .
$$

Now, making the change of variable $u=v^{2}$ in the above integral, we have that

$$
£_{\beta \beta}=\frac{h(\alpha)}{\alpha \beta^{2} \sqrt{2 \pi}},
$$

where

$$
h(\alpha)=\int_{0}^{\infty} \frac{1}{v^{2}\left(1+v^{2}\right)} \exp \left\{-\frac{1}{2 \alpha^{2}}\left(v-v^{-1}\right)^{2}\right\} \mathrm{d} v .
$$

Making the change of variable $u=v-v^{-1}$ it follows that

$$
\begin{aligned}
h(\alpha)= & \frac{1}{2} \int_{-\infty}^{\infty} \frac{u^{2}+2-u \sqrt{u^{2}+4}}{u^{2}+4} \exp \left(-\frac{u^{2}}{2 \alpha^{2}}\right) \mathrm{d} u \\
= & \frac{1}{2} \int_{-\infty}^{\infty} \exp \left(-\frac{u^{2}}{2 \alpha^{2}}\right) \mathrm{d} u-\int_{-\infty}^{\infty} \frac{1}{u^{2}+4} \exp \left(-\frac{u^{2}}{2 \alpha^{2}}\right) \mathrm{d} u \\
& -\frac{1}{2} \int_{-\infty}^{\infty} \frac{u}{\sqrt{u^{2}+4}} \exp \left(-\frac{u^{2}}{2 \alpha^{2}}\right) \mathrm{d} u \\
= & \alpha \sqrt{\frac{\pi}{2}}-\int_{-\infty}^{\infty} \frac{1}{u^{2}+4} \exp \left(-\frac{u^{2}}{2 \alpha^{2}}\right) \mathrm{d} u .
\end{aligned}
$$

Let

$$
g(\xi)=\int_{-\infty}^{\infty} \frac{1}{u^{2}+4} \exp \left(-\frac{\xi^{2} u^{2}}{2}\right) \mathrm{d} u,
$$

where $\xi=1 / \alpha$ for notational convenience. Then,

$$
\begin{aligned}
g^{\prime}(\xi) & =-\xi \int_{-\infty}^{\infty} \frac{u^{2}}{u^{2}+4} \exp \left(-\frac{\xi^{2} u^{2}}{2}\right) \mathrm{d} u \\
& =-t \int_{-\infty}^{\infty} \exp \left(-\frac{\xi^{2} u^{2}}{2}\right) \mathrm{d} u+4 t \int_{-\infty}^{\infty} \frac{1}{u^{2}+4} \exp \left(-\frac{\xi^{2} u^{2}}{2}\right) \mathrm{d} u \\
& =-\sqrt{2 \pi}+4 \operatorname{tg}(\xi)
\end{aligned}
$$


that is,

$$
g^{\prime}(\xi)=4 \operatorname{tg}(\xi)-\sqrt{2 \pi} .
$$

The solution of the differential equation (.1) is given by

$$
g(\xi)=\mathrm{e}^{2 \xi^{2}}[C-\pi \Phi(2 \xi)] .
$$

Notice that $g(0)=\pi / 2$ and then $C=\pi$. Thus

$$
\int_{-\infty}^{\infty} \frac{1}{u^{2}+4} \exp \left(-\frac{\xi^{2} u^{2}}{2}\right) \mathrm{d} u=g(1 / \alpha)=\pi \mathrm{e}^{2 / \alpha^{2}}[1-\pi \Phi(2 / \alpha)] .
$$

Therefore, we have that

$$
h(\alpha)=\alpha\left(\frac{\pi}{2}\right)^{1 / 2}-\pi \mathrm{e}^{2 / \alpha^{2}}\left[1-\Phi\left(\frac{2}{\alpha}\right)\right]
$$

and hence

$$
K_{\beta \beta}=\frac{1+\alpha(2 \pi)^{-1 / 2} h(\alpha)}{(\alpha \beta)^{2}} .
$$

By a similar algebraic development (but of independent interest), we can show that

$$
\int_{0}^{\infty} \frac{1}{v^{2}\left(1+v^{2}\right)^{2}} \exp \left\{-\frac{1}{2 \alpha^{2}}\left(v-v^{-1}\right)^{2}\right\} \mathrm{d} v=\alpha\left(\frac{\pi}{2}\right)^{1 / 2}-\frac{3 \pi}{2} \mathrm{e}^{2 / \alpha^{2}}\left[1-\Phi\left(\frac{2}{\alpha}\right)\right] .
$$

Finally, the R functions used in this paper to compute $h(\alpha)$ and $h_{1}(\alpha)$ in equations (2.1) and (3.1), respectively, are given by

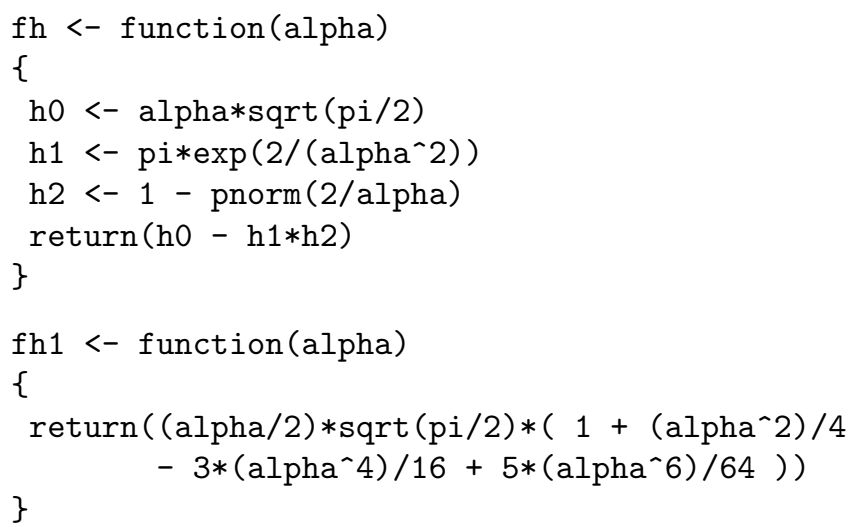

\section{References}

[1] Abramowitz, M., Stegun, I.A. (1970). Handbook of Mathematical Functions. National Bureau of Standards, Washington.

[2] Achcar, J.A., Moala, F.A. (2010). Use of MCMC methods to obtain Bayesian inferences for the Birnbaum-Saunders distribution in the presence of censored data and covariates. Advances and Applications in Statistics 17, 1-27.

[3] Aslam, M., Jun, C.H., Ahmad, M. (2011). New acceptance sampling plans based on life tests for Birnbaum-Saunders distributions. Journal of Statistical Computation and Simulation 81, 461-470.

[4] Bhatti, C.R. (2010). The Birnbaum-Saunders autoregressive conditional duration model. Mathematics and Computers in Simulation 80, 2062-2078.

[5] Birnbaum, Z.W., Saunders, S.C. (1969a). A new family of life distributions. Journal of Applied Probability 6, 319-327.

[6] Birnbaum, Z.W., Saunders, S.C. (1969b). Estimation for a family of life distributions with applications to fatigue. Journal of Applied Probability 6, 328-347. 
[7] Castillo, N.O., Gomez, H.W., Bolfarine, H. (2011). Epsilon Birnbaum-Saunders distribution family: properties and inference. Statistical Papers 52, 871-883.

[8] Cordeiro, G.M., Lemonte, A.J. (2011). The $\beta$-Birnbaum-Saunders distribution: An improved distribution for fatigue life modeling. Computational Statistics and Data Analysis 55, 1445-1461.

[9] Cordeiro, G.M., Lemonte, A.J., Ortega, E.M.M. (2013). An extended fatigue life distribution. Statistics 47, 626-653.

[10] Cox, D.R., Reid, N. (1987). Parameter orthogonality and approximate conditional inference (with discussion). Journal of the Royal Statistical Society B 40, 1-39.

[11] Desmond, A.F. (1985). Stochastic models of failure in random environments. Canadian Journal of Statistics 13, 171-183.

[12] Desmond, A.F. (1986). On the relationship between two fatigue-life models. IEEE Transactions on Reliability 35, 167-169.

[13] Díaz-García, J.A., Leiva, V. (2005). A new family of life distributions based on the elliptically contoured distributions. Journal of Statistical Planning and Inference 128, 445-457.

[14] Engelhardt, M., Bain, L.J., Wright, F.T. (1981). Inferences on the parameters of the Birnbaum-Saunders fatigue life distribution based on maximum likelihood estimation. Technometrics 23, 251-256.

[15] Gómes, H.W., Olivares-Pacheco, J.F., Bolfarine, H. (2009). An extension of the generalized BirnbaumSaunders distribution. Statistics and Probability Letters 79, 331-338.

[16] Guiraud, P., Leiva, V., Fierro, R. (2009). A non-central version of the Birnbaum-Saunders distribution for reliability analysis. IEEE Transactions on Reliability 58, 152-160.

[17] Kundu, D., Balakrishnan, N., Jamalizadeh, A. (2010). Bivariate Birnbaum-Saunders distribution and associated inference. Journal of Multivariate Analysis 101, 113-125.

[18] Kundu, D., Balakrishnan, N., Jamalizadeh, A. (2013). Generalized multivariate Birnbaum-Saunders distributions and related inferenctial issues. Journal of Multivariate Analysis 116, 230-244.

[19] Kundu, D., Kannan, N., Balakrishnan, N. (2008). On the hazard function of Birnbaum-Saunders distribution and associated inference. Computational Statistics and Data Analysis 52, 2692-2702.

[20] Leiva, V., Barros, M., Paula, G.A., Sanhueza, A. (2008). Generalized Birnbaum-Saunders distributions applied to air pollutant concentration. Environmetrics 19, 235-249 .

[21] Lemonte, A.J. (2013). A new extension of the Birnbaum-Saunders distribution. Brazilian Journal of Probability and Statistics 27, 133-149.

[22] Lemonte, A.J., Cribari-Neto, F., Vasconcellos, K.L.P. (2007). Improved statistical inference for the two-parameter Birnbaum-Saunders distribution. Computational Statistics and Data Analysis 51, 46564681.

[23] Lemonte, A.J., Ferrari, S.L.P. (2011). Testing hypotheses in the Birnbaum-Saunders distribution under type-II censored samples. Computational Statistics and Data Analysis 55, 2388-2399.

[24] Lemonte, A.J., Simas, A.B., Cribari-Neto, F. (2008). Bootstrap-based improved estimators for the twoparameter Birnbaum-Saunders distribution. Journal of Statistical Computation and Simulation 78, 3749.

[25] Marshall, A.W., Olkin, I. (2007). Life Distributions. Structure of Nonparametric, Semiparametric and Parametric Families. Springer, New York.

[26] Meintanis, S.G. (2010). Inference procedures for the Birnbaum-Saunders distribution and its generalizations. Computational Statistics and Data Analysis 54, 367-373.

[27] Nikulin, M.S. and Tahir, R. (2010). Application of Sedy akin's model and Birnbaum-Saunders family for statistical analysis of redundant systems with one warm stand-by unit. Veroyatnost'i Statistika 17 , 155-171.

[28] Ng, H.K.T., Kundu, D., Balakrishnan, N. (2003). Modifiel moment estimation for the two-parameter Birnbaum-Saunders distribution. Computational Statistics \& Data Analysis 43, 283-298.

[29] Owen, W.J. (2006). A new three-parameter extension to the Birnbaum-Saunders distribution. IEEE Transactions on Reliability 55, 475-479.

[30] Podlaski, R. (2008). Characterization of diameter distribution data in near-natural forests using the Birnbaum-Saunders distribution. Canadian Journal of Forest Research 38, 518-527. 
[31] R Development Core Team (2012). R: A Language and Environment for Statistical Computing. R Foundation for Statistical Computing, Vienna, Austria.

[32] Saunders, S.C. (2007). Reliability, Life Testing, and Prediction of Service Lives. Springer, New York.

[33] Vilca, F., Sanhueza, A., Leiva, V. and Christakos, G. (2010). An extended Birnbaum-Saunders model and its application in the study of environmental quality in Santiago, Chile. Stochastic Environmental Research and Risk Assessment 24, 771-782.

[34] Wang, B.X. (2012). Generalized interval estimation for the Birnbaum-Saunders distribution. Computational Statistics and Data Analysis 56, 4320-4326. 